\title{
Effective low-noise technologies for the production of pile works in construction
}

\author{
Vladimir Chernyuk ${ }^{1 *}$, Sergey Semenyuk ${ }^{1}$, and Vitaly Yuskovich $^{1}$ \\ ${ }^{1}$ Brest State Technical University, Belarus
}

\begin{abstract}
The article describes the environmental features of the production of pile works (noise, vibration), including by driving methods. They are the most unfavorable, the value of which exceeds the maximum permissible norms for noise and vibration by more than 2 times, and they are constantly growing from year to year, and this is the cause of many different diseases of people, including mental and neuralgic ones. In this regard, individual and collective methods of dealing with noise are recommended, as well as silent and low-noise methods of piling work, protected by the a.s.USSR and patents of the Republic of Belarus for inventions and utility models.
\end{abstract}

\section{Introduction}

Environmental protection and the protection of workers' health are two of the most important problems of our time in all countries of the world. An integral part of them, along with others, is the fight against noise and vibration. The noise level at work and at home can reach over $100 \mathrm{~dB}$ and exceed 1.5-2 times the maximum permissible noise standards and it is constantly growing from year to year by $1.5-2 \mathrm{~dB}$, and this is the cause of many diseases of people, including neuralgic, which make up almost $80 \%$ of the total number of people with this disease.

Noise in industrial and residential construction is particularly harmful, as it occurs, as a rule, in areas with high population density, which is confirmed by the data given in [1-3]. At the same time, about $41 \%$ of the complaints of the population account for noise and vibration during the operation of diesel hammers, $12 \%$ - for the operation of vibratory hammers, copers and vibratory loaders, $17 \%$ - for the operation of jackhammers of drilling devices, $7 \%$ - for the operation of compressors, $23 \%$ - for the operation of other construction machines (cranes, excavators, cars, etc.). Approximately half of the complaints of the population are due to noise and vibration that occur during construction and installation works. Basically, these are psychological reasons - interference with sleep, rest, work, conducting training sessions, conducting physical culture events. About $2 / 3$ of the complaints of the population $(65 \%)$ are filed with the courts for compensation for moral damage caused to the health of the population, and another $1 / 3$ - for compensation for material damage caused to residential buildings and buildings adjacent to construction sites where construction work is underway. This is mainly due not only to noise, but also to

\footnotetext{
* Corresponding author: tsp@,bstu.by
} 
vibration associated with the installation of pile foundations and the operation of pilecutting equipment.

In some countries of Western Europe, for example, France, Great Britain, Asia and America, Japan, Singapore, New York, where there are many high-rise buildings, the production of piling works by hammering and other shock methods is prohibited by law due to the high level of vibration and excessive sound pressure that occurs during the operation of piling equipment, as well as the need to protect and preserve historical values, monuments, buildings and structures, as well as human health. This has prompted many construction firms to switch to other shock-free, silent and low-noise methods of pile foundations, technologies and types of piles - from hammered to stuffed and bored (Strauss piles, Benoto, Franks, vibro-stamped, vibro-punched, non-hammered, frequency-rammed and others), and firms producing pile hammers and copra, to rebuild to improve and produce machines with their improved technical and technological characteristics, with sound-proofing casings, rubber penetrations, anti-vibration devices and devices (many firms in Germany, USA, England, Sweden and Japan).

\section{Noise and vibration problems}

As numerous researchers have shown, noise and vibration to a greater or lesser extent can temporarily activate or permanently suppress certain psychological processes in the human body and lead to various neuralgic and mental illnesses. Physiological consequences can manifest themselves in the form of hearing impairment and other analyzers, for example, the vestibular apparatus that coordinates the functions of the cerebral cortex, nervous and digestive systems, circulatory system, heart. In addition, noise affects carbohydrate, fat and protein metabolism in the body.

Vibration, in turn, affects the central nervous system, gastrointestinal tract, balance organs (vestibular apparatus), causes dizziness, numbness of the limbs, joint disease. Prolonged exposure to vibration leads to occupational disease - vibration disease, the effective treatment of which is possible only in the early stages, and the restoration of impaired functions is extremely slow (and not always), and under certain conditions irreversible processes may occur in the body, accompanied by complete loss of working capacity and the onset of disability.

The greatest level of noise and vibration, and these are the accompanying adverse environmental factors affecting a person in construction, as the analysis shows, are created by dynamically operating machines and mechanisms, equipment and tools of impact action.

The most troublesome in terms of noise, sound, vibration and vibrations, the degree of impact on a person and the order of importance are pile-driving tools, especially diesel hammers (any, both rod and tubular), copers and coper units on any kind (rail, tractor, automobile, excavator, bridge type), then vibratory lots and vibratory loaders, rolling stock of railway transport, as well as buses, trucks, passenger cars and special machines, cranes, excavators and other earthmoving equipment, bulldozers, concrete mixers, concrete pavers and other construction equipment.

For example, when a pile driver equipped with a diesel hammer is working, the maximum sound pressure level exceeds $100 \mathrm{~dB}$ at a distance from it, and in a radius of 100 $\mathrm{m}$ (and this is a microdistrict) reaches $80-100 \mathrm{~dB}$, which has a harmful effect on the health of the worker and others, increases their fatigue and, accordingly, reduces labor productivity.

The sound pressure levels measured by the authors with a noise meter ("Octave 101A") for different methods of pile immersion gave the following results:

- pile driving with diesel hammers - 100-120 dB;

- vibration loading of piles by vibration loaders - 80-100 dB; 
- vibro-hammering of piles with vibro-hammers - 80-110 dB.

Similar unsatisfactory results were obtained by vibration measurements with a vibrometer ("Octave - 101A").

At the same time, the maximum permissible sound pressure levels for the main most typical types of work and workplaces are in octave bands with average geometric frequencies 31,$5 ; 63 ; 125 ; 250 ; 500 ; 1000 ; 2000 ; 4000 ; 8000 \mathrm{~Hz}$ according to the SanPiN 22.4/2.18.1032-2002 They are shown in Figures 1, 2, since human hearing organs perceive sound vibrations in the frequency range from 16 to $20000 \mathrm{~Hz}$, and the zone of greatest hearing sensitivity is in the region of $50-5000 \mathrm{~Hz}$, which is located in the octave bands. Vibrations with a frequency of up to $16 \mathrm{~Hz}$ (infrasound) and above $20000 \mathrm{~Hz}$ (ultrasound) are not perceived by human hearing organs. However, this does not mean that the effect of infrasound and ultrasound above the maximum permissible standards is not harmful to humans.

Permissible sound levels according to SN 3077-84 in wards of hospitals and sanatoriums, residential and dormitory houses, children's institutions, schools, universities, polyclinics, rest homes, libraries, shops, automobile and railway stations, reception points are given in [3]. They are approximately $20-40 \mathrm{~dB}$ at night and 30-70 dB during the day, and this is 1.5-2 times lower than the actual values when driving piles.

\section{Noise abatement methods}

In general, the means of protecting a person from noise, including pile noise, can be divided into individual and collective. The first include headphones, anti-noise earbuds, headsets.

Collective means of protection include:

- sound insulation - fences, consoles, cabins, enclosures, fences and "green walls" that prevent the spread of noise from one room to another or in the same room;

- sound absorption - the ability of a material or structure to absorb the energy of sound waves. These include facings, piece sound absorbers, sound-absorbing barriers. The latter are divided into 4 classes: fibrous-porous (felt, cotton wool, acoustic plaster, ultrathin glass or basalt fiber), membrane (PVC and other tiles, thin sheets of plywood or metal on a crate), resonant (special designs based on the acoustic properties of the resonator), combined (devices using previous materials);

- silencers - adsorption, active, reactive and combined of various types.

Such collective means of protection can be much more effective in the production of pile works as:

- elimination or reduction of noise and vibration directly in the sources of their occurrence, i.e. in pile-cutting installations, can be much more effective in the production of pile works;

- localization of noise and vibration sources in pile-loading equipment;

- rational placement of pile-cutting equipment on the construction site;

- acoustic treatment of auxiliary rooms for maintenance personnel;

- introduction of silent and low-noise technologies, technological processes and equipment.

We will focus on the latter, as the most promising and effective way to combat noise, separately.

Silent ones include the technology of installing piles in large-diameter wells previously drilled in dense, durable and frozen soils (large diagonals of the cross-section of the pile), when it is impossible to hammer the pile and where a lot of noise is made when driving, followed by filling the sinuses with a ground solution-sludge. The method relates to drilling and lowering piles, is applicable and only possible in conditions of permafrost distribution (Norilsk, Vorkuta, Yakutia and other regions) in the Far North of the Russian Federation 
and in areas of strong and rocky soils, including in the Republic of Belarus, in which it is simply impossible to hammer piles.

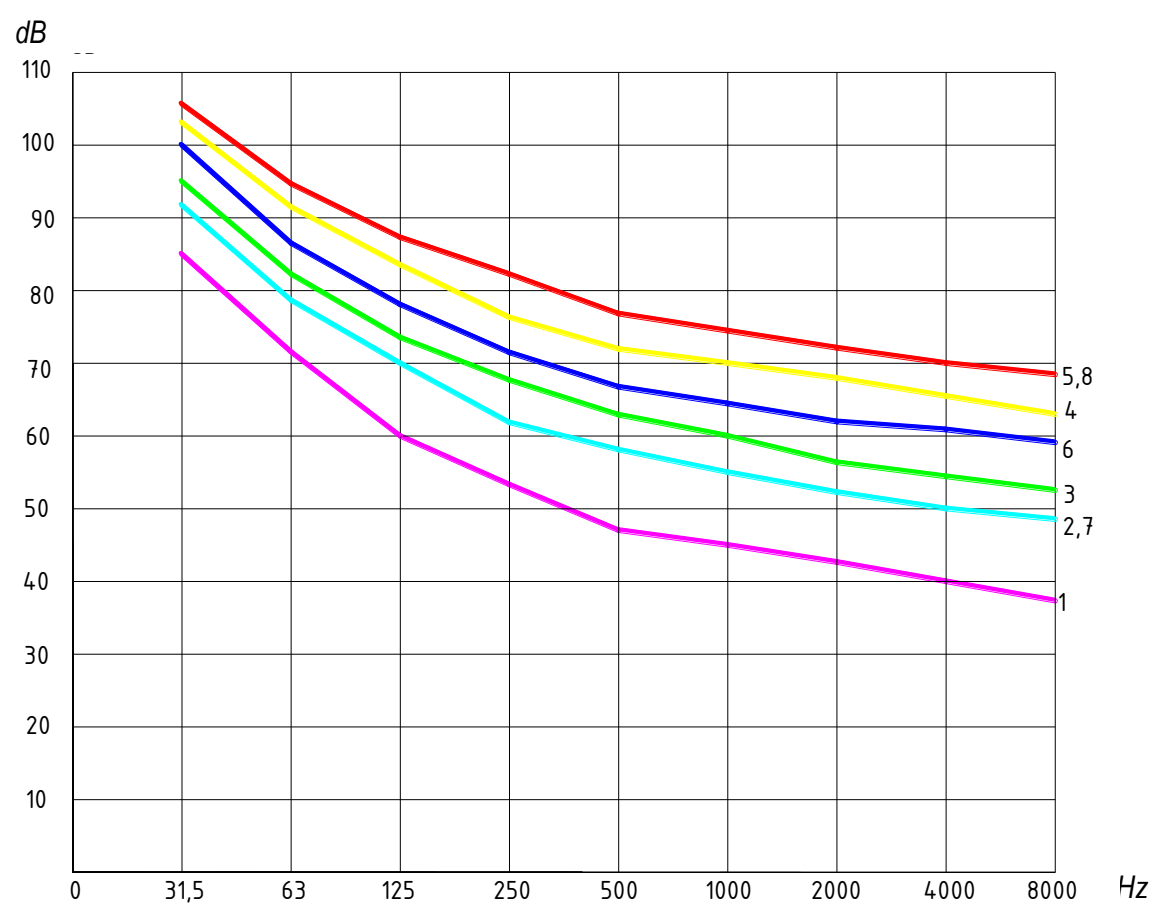

1 - creative activity, managerial work with increased requirements, design work, study, teaching, medical activity, etc.; 2 - concentrated, highly qualified and administrative and managerial work; 3 - operator and dispatching work; 4 - concentrated and supervisory work;

5 - work at permanent places (except for the above); 6 - jobs on the rolling stock of railway transport, drivers and maintenance personnel of trucks; 7 - the same, passenger transport;

8 - the same as the personnel of self-propelled chassis tractors, trailed and mounted agricultural machines, road construction and other similar machines

Fig. 1. Maximum permissible sound pressure levels for the main typical types of work and workplaces $(\mathrm{dB})$ in octave bands with average geometric frequencies $(\mathrm{Hz})$.

The method of driving piles into pre-drilled wells of small diameter (leader wells), the diameter of which is equal to or less than $5 \mathrm{~cm}$ of the size of the square section of the pile, is considered low-noise. The conditions for using the method are the same as those of silent ones, but the sound pressure level is reduced by half compared to driven piles.

The low- and noiseless technologies of pile immersion should also include their hydraulic immersion by washing the soil with water under the piles, as well as their immersion with coating and in thixotropic clay jackets. In these methods, the sinking force of the piles is reduced by $50-70 \%$, which also helps to reduce noise and vibration. In the development of these methods, the authors of the BSTU in different years have proposed a number of technical developments for effective designs of driven piles and methods of their immersion by hydraulic and other methods, as well as coatings protected by a. s. USSR (№№779507, 779508, 881201, 887725, 891840, 947278, 912447, 962454, 1004539, 1032102, 1135843, 1153010, 1157164, 1488500), patents of the Republic of Belarus for inventions and utility models published in $[4,5]$. 
$d B$

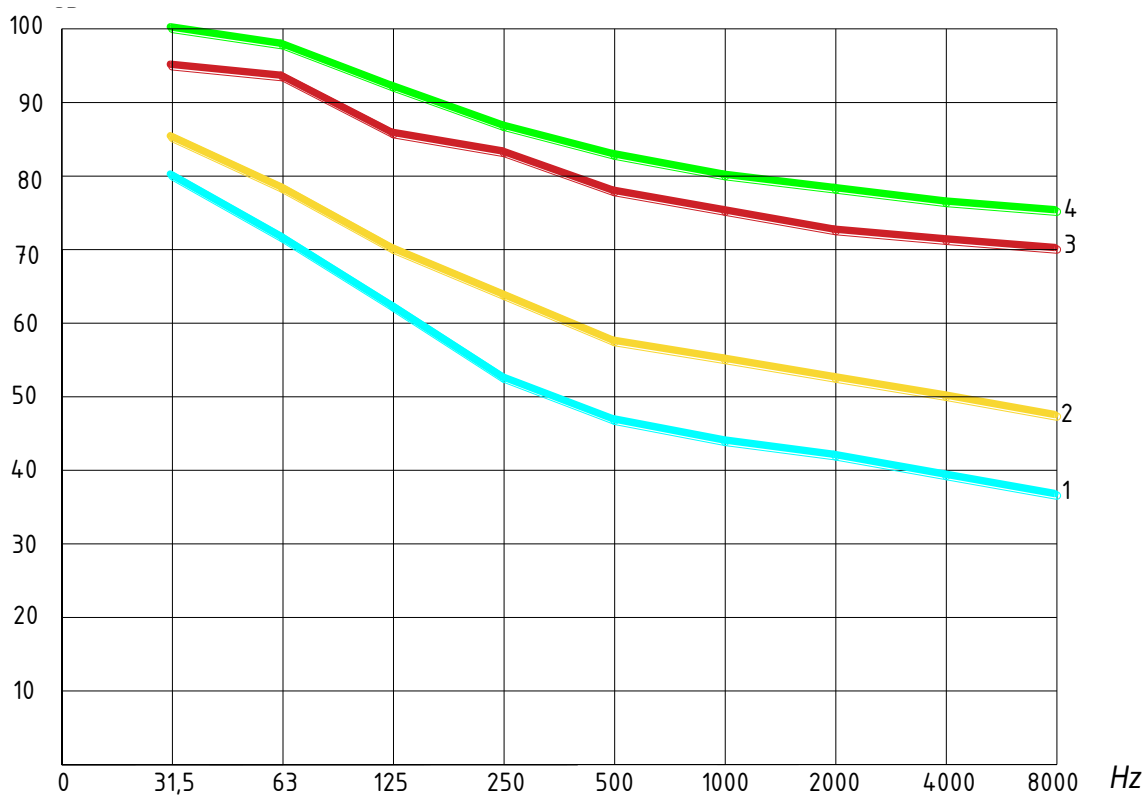

1 - rooms of design bureaus, laboratories for processing experimental data;

2 - control rooms, workrooms; 3 - laboratory rooms for experimental work; 4 - permanent workplaces and work areas in production facilities and on the territory of enterprises

Fig. 2. Permissible sound pressure levels in workplaces, industrial premises and on the territory of enterprises $(\mathrm{dB})$ in octave bands with average geometric frequencies $(\mathrm{Hz})$.

Washing the soil with water under piles with high-pressure pumps or gravity is used to facilitate their immersion and reduce the noise level, under shell piles, especially of large dimensions (cross section and length), in sandy (incoherent) and poorly connected (loamy and clay) soils, as well as with a large immersion depth and insufficient immersion capacity (power) of pile-breaking or vibration-loading equipment or mechanism. The method is applicable if it cannot cause subsidence of buildings and structures located in the neighborhood.

The essence of the washout lies in the fact that water is brought to the tip of the pile by one or several pipes fixed on the sides, the soil is washed away with it, and the pile is immersed in the ground under the action of its own weight and the weight of the hammer installed on it or priming. At the last stage of immersion, the washing is stopped and the pile is finished off in the usual way. Hanging piles by the method of hydraulic washing should be immersed carefully, since this breaks the adhesion of the side surface of the pile with the ground. The effect of hydraulic washing is that under the action of water pressure, the drag of the soil is reduced due to washing. In addition, the water flow, rising along the side surface of the pile, erodes the soil and weighs its particles. As a result, the resistance of the soil decreases and the required sinking force of the pile decreases.

It is known that the proportion of soil resistance under the tip of the pile is more than half of the total resistance to immersion, the rest (about 50\%) is the friction force on the side surface of the pile. Their ratio depends on the length and dimensions of the crosssection of the pile (the longer the pile, the smaller the proportion of the drag of the soil, and vice versa), the physical and mechanical properties of the soil and other parameters. In order to reduce the friction (adhesion) of the pile on the ground during immersion, it is also advisable to apply a coating of the side surface of the trunk with materials with high anti- 
friction properties and a low coefficient of friction. Such materials are clay thixotropic solutions and pastes, carbamide, furfurolaniline, polyacrylamide and epoxy resins and coatings, as well as vora as a lubricant. In this direction, the TSP department has created a number of progressive developments.

Several simple and effective hydraulic washing and self-lubricating pile structures are shown in Figure 3. The reduction of the frontal (under the end of the trunk) and lateral (along the outer surface of the trunk) pile resistances during its immersion occurs due to the forced supply of water or lubricant to the friction zone and the destruction of the soil under the end and on the side surface of the pile.

a)
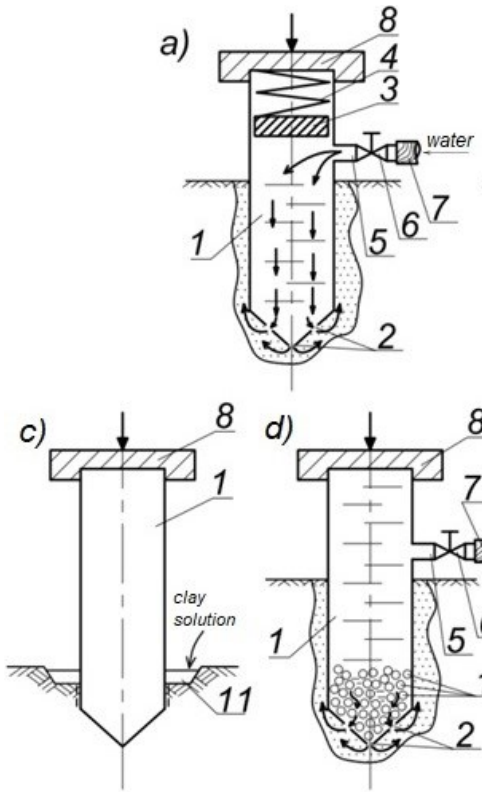

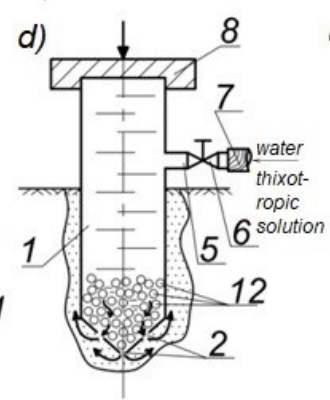

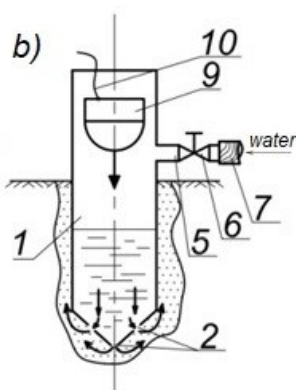

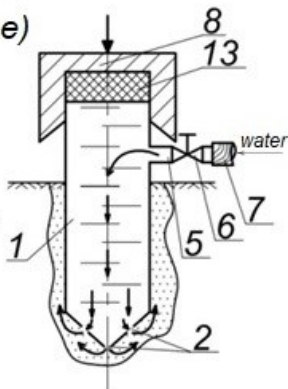

1 - pile;

2 - through holes;

3 - solid disc;

4 - compression spring;

5 - branch pipe;

6 - valve;

7 - hose;

8 - headrest;

9 - suspended load;

10 - flexible rod;

11 - pit with ticsotropny solution;

12 - large-block rounded inclusions (boulders, pebbles);

13 - elastic shock-absorbing rubber gasket

a) - with a spring-loaded disc; b) - with a suspended load; c) - with a thixotropic coating;

d) - with large-block inclusions; e) with a shock-absorbing gasket

Fig. 3. Efficient designs of hydraulic washing (a, b, d, e) and lubricated piles (c, d).

Figure 3, a (RB patent for utility model No. 3603) shows a driven pile with a springloaded disc. When such a pile is immersed in the ground in any way, preferably hammered with a diesel hammer, sharp vibrations of a solid disk, spring-loaded from above by a compression spring, will occur in the trunk cavity. When striking, the pile abruptly moves down, the solid disk moves up, compressing the spring and opening the water supply pipe. After removing the force of the diesel hammer, the disk under the action of a compressed spring rushes sharply down, creating a hydraulic shock in the barrel cavity, partially or completely blocking the nozzle and cutting off the water. Due to the hydraulic shock, the water rushes down sharply under increased pressure to the through holes and exits to the outer surface of the tip, lubricating it with water and reducing the resistance to immersion of the pile under the end face and friction along the side surface of the barrel.

As a result, the rate of immersion of the pile increases, the energy intensity and noise during its immersion decrease, the number of impacts on driving into the ground decreases with a simple, efficient and reliable design.

In the second design (Fig. 3, b), protected by the patent of the Republic of Belarus for invention No. 6032, water or a portion of a fixing or lubricating solution (for example, silicate or thixotropic) is poured into the plane of the pile, which is then rammed and 
squeezed through through holes under the bottom of the pile and to the lateral outer surface of the trunk, helping to reduce the energy intensity of immersion, noise and vibration, fixing the soil and increasing the bearing capacity on the ground of the base.

In the third design (Fig. 3, c, RB patent for utility model No. 8601), a lubricating substance (clay solution) is fed into the pit to the places of immersion of the pile. As a result, this substance lubricates the outer side surface of the pile, which reduces the friction and adhesion of the soil with the trunk, and as a result also reduces the energy intensity of immersion, noise and the number of impacts per dive.

In the fourth driven pile, protected by three patents of the Republic of Belarus for utility models No. 5228, 7573 and 9781 (Fig. 3, d), the pile cavity is filled with water or other lubricating substance, for example, clay solution, and additionally filled with coarsegrained material (pebbles, gravel, small boulders). When driving a pile with a diesel hammer or vibration immersion, the large-block material in the cavity begins to oscillate, bounce, settle, creating many small, medium and large hydraulic shocks, and this, in turn, leads to the same consequences - improving the permeability of water or lubricant through the holes under the bottom of the pile and to the side surface of the trunk, which also gives its positive results.

In the driving pile shown in Figure 3, e (patent for invention RB No. 10518), the pile head is of interest, in the box-shaped housing of which a shock-absorbing rubber gasket is mounted, which helps to improve the passage of water through the cavity of the pile to through holes and, as a result, better lubrication of the tip and side surface of the trunk, erosion of the soil by water, reduction of frontal and lateral resistance of the soil, reduction of noise during driving. Of particular interest is such a driven pile according to the patent of the Republic of Belarus for utility model No. 6882.

\section{Conclusion}

The use of individual and collective means of protection against noise and vibration, as well as silent and low-noise methods of pile work, hydraulic washing of soil with water under piles, coating their side surface with antifriction materials and lubricants allows to reduce the noise level when sinking piles in a hammered way to normal limits, not higher than permissible values, and also reduce the risk of occupational diseases at work.

\section{References}

1. Krechin, A.S. Resource-saving foundations on rural construction sites / A. S. Krechin, V.P. Chernyuk, P.V. Shvedovsky, etc- - Chisinau, Kartya Moldovenyaske, 1990. - 247 s.

2. Chernyuk, V.P. Calculation, design and arrangement of pile foundations/ V.P.Chernyuk, P.S. Poita. - Brest, Obltypografiya, 1998. -216 s.

3. Chernyuk, V.P. Noise reduction during the operation of piling equipment/ V.P. Chernyuk, P.P. Ivasyuk, G.E. Rebrov // Vestnik BrGTU "Water management construction, heat power engineering and Geoecology", No. 8, - Brest, BrSTU, 2015. s. 124-128.

4. Chernyuk, V.P. Production of pile works in special conditions / V.P. Chernyuk, P.P. Ivasyuk. - Germany, LAPLAMBERT Academic Publishing, 2016 - 196 s.

5. Chernyuk, V.P. Technology of pile works in special conditions of construction / V.P. Chernyuk, E.I. Shlyakhova // Textbook. - M.: LLC "Rusains. 2019”. - 241 s. 